\title{
Les applications thérapeutiques de la radioactivité
}

\author{
Roland HusTINX \\ Service de Médecine nucléaire \& Imagerie oncologique \\ CHU de Liège \\ GIGA-CRC in vivo Imaging \\ Université de Liège
}

Les applications thérapeutiques de la radioactivité relèvent de deux disciplines, la radiothérapie et la médecine nucléaire. Les techniques proposées sont très diverses, certaines inchangées depuis des décennies, d'autres tentant d'appliquer des concepts parmi les plus novateurs de la médecine moderne. Si le nombre de total des patients concernés par ces techniques peut paraître limité au regard des autres modalités thérapeutiques (pharmacologiques, chirurgicales, etc.), le domaine n'en est pas moins extrêmement actif et porteur d'espoir dans un nombre croissant de pathologies.

\section{Aspects physiques}

Les applications thérapeutiques de la radioactivité tirent profit des caractéristiques physiques du rayonnement émis. Le paradigme est inverse à celui qui sous-tend le choix du radio-isotope pour les applications diagnostiques. Concernant ces dernières, il s'agit de capter le rayonnement émis par le patient, et de le transformer en information utile, souvent sous la forme d'images. On souhaite donc un rayonnement dont le parcours est suffisant pour être enregistré par les détecteurs, dont l'énergie est propice à l'imagerie, c'est à dire permettant un enregistrement efficace par ces détecteurs, et une période physique relativement courte de manière à limiter l'exposition du patient. On fera appel à des émetteurs gamma ou à des émetteurs de positons, qui donneront eux-mêmes naissance à des photons gamma, après désintégration. S'agissant des applications thérapeutiques, il s'agit d'induire une mort cellulaire. On souhaite donc typiquement un rayonnement de plus haute énergie, avec un parcours aussi court que possible, et une période physique propice à un débit de dose aussi efficace que possible sur le plan biologique. On se tournera donc vers des isotopes émetteurs de rayonnement beta ou alpha, dont le parcours dans les tissus vivants est limité à quelques mm et quelques dizaines de $\mu \mathrm{m}$, respectivement.

Le principe de base consiste à introduire dans un tissu cible, ou à son voisinage immédiat, une source radioactive de manière à l'irradier de façon aussi ciblée que possible, afin d'épargner les tissus sains. On distingue deux grandes méthodes. La première, appelée curiethérapie ou brachythérapie, utilise des sources radioactives scellées, se présentant sous la forme de grains ou de bâtonnets, et qui sont introduites dans ou au voisinage de la tumeur. Les applications les plus fréquentes concernent les néoplasies prostatiques et du col utérin. Ce traitement est réalisé par le radiothérapeute. La seconde modalité utilise également des sources radioactives, mais sous forme non scellée, c'est-à-dire dispersible et souvent métabolisable. On parle ici de radiothérapie métabolique, ou de radiothérapie interne vectorisée, qui est l'expression officielle préconisée en France. Nous nous concentrerons sur cette modalité pour la suite de cette revue. 


\section{Aspects cliniques}

Les premiers traitements ont été réalisés en 1938 par JH Lawrence, par des administrations orales répétées d'une solution contenant du Phosphore-32, à des patients souffrant de leucémies et qui présentaient une polycythémie (augmentation anormale du nombre d'érythrocytes dans le sang). Le

${ }^{32} \mathrm{P}$ est un émetteur beta qui s'accumule dans les globules rouges, entrainant leur destruction. Ce traitement n'est plus indiqué mais il présentait déjà les éléments qui sous-tendent la radiothérapie métabolique, à savoir une irradiation ciblée après introduction du radio-isotope dans l'organisme. L'administration du médicament radioactif peut s'effectuer de diverses manières : intracavitaire, par voie orale, intraveineuse, voire intra-artérielle. Dans le premier cas, on peut par exemple injecter une solution d'Yttrium-90 ou d'Erbium-169, à l'intérieur d'une articulation (genou, poignet) afin de détruire la membrane synoviale enflammée, qui ne répond pas aux traitements classiques. On parlera de radiosynoviorthèse.

L'administration orale est possible lorsque le radionucléide est bien absorbé par le tube digestif, et que sa biodistribution le mène naturellement dans un tissu cible. C'est le cas du traitement qui reste le plus fréquent en routine clinique, l'iode-131 dans les pathologies thyroïdiennes. En effet, $1^{131}$ I administré sous la forme d'une gélule, est totalement absorbé et se retrouve donc dans le flux sanguin. Il se concentre ensuite dans les zones actives de la thyroïde, ainsi que, une dans une moindre mesure, dans les glandes salivaires, le surplus étant éliminé par voie rénale. Les cellules thyroïdiennes ne font aucune différence entre l'iode radioactif et l'iode froid, leur biodistribution est rigoureusement identique. Cette approche est utile dans certaines formes d'hyperthyroïdie telles que l'adénome toxique ou la maladie de Basedow. Dans les cancers thyroïdiens bien différenciés, c'est à dire qui conservent la capacité de concentrer l'iode, le but est de détruire toutes les cellules thyroïdiennes, saines et malignes, et l'activité administrée est dès lors beaucoup plus élevée.

L'administration intraveineuse peut s'envisager lorsque d'une part, de manière similaire à l'iode, le radio-isotope se distribue de façon spécifique, ou à tout le moins préférentielle, dans le tissu cible et d'autre part l'absorption digestive est insuffisante. On peut citer le dichlorure de radium-223 (Xofigo ${ }^{\circledR}$ ) dont l'accumulation dans le squelette est proportionnelle au remodelage osseux. Cet émetteur alpha se concentre dans les métastases osseuses dites ostéocondensantes, avec un effet à la fois antalgique et tumoricide (figure 1).

Il est associé à une augmentation de la survie dans des populations sélectionnées de patients souffrants de métastases osseuses d'un cancer de la prostate (1). Le plus souvent cependant, la voie intraveineuse implique la vectorisation du radio-isotope, c'est à dire son couplage à un vecteur qui le mènera dans le tissu cible. Ce vecteur peut être un anticorps monoclonal ou un peptide, par exemple. Dans certaines situations, on évoquera un concept ancien, remis au goût du jour, sous le terme «theranostique ». Le principe est simple : une méthode d'imagerie, à l'aide d'un radiotraceur, permet de définir la cible puis dans un second temps de la traiter via l'administration d'un radiopharmaceutique identique, ou similaire, couplé cette fois à un isotope destructeur, émetteur alpha ou beta. Ainsi, les tumeurs neuroendocrines surexpriment à leur surface cellulaire les récepteurs à la somatostatine. 


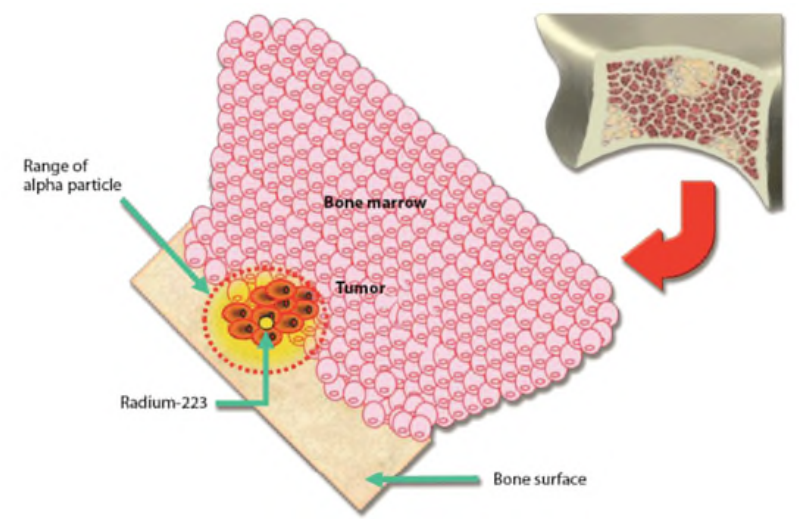

Figure 1 : Le Radium-223 émet un rayonnement alpha, dont le parcours est très limité. Il délivre ainsi une grande quantité d'énergie sur une distance très courte, concentrant ses effets sur les cellules métastatiques osseuses et irradiant beaucoup plus faiblement les cellules de la moelle osseuse.

Si l'on administre au patient un peptide, analogue de la somatostatine (DOTATATE) marqué au ${ }^{68} \mathrm{Ga}$, il sera possible de réaliser une imagerie PET/CT et d'obtenir une «cartographie » de l'expression des récepteurs sur les lésions. Si la situation est favorable, on administre dans un second temps le même peptide, marqué cette fois avec du ${ }^{177} \mathrm{Lu}$, avec pour but de détruire toutes les cellules surexprimant les récepteurs à la somatostatine (figure 2) (2).

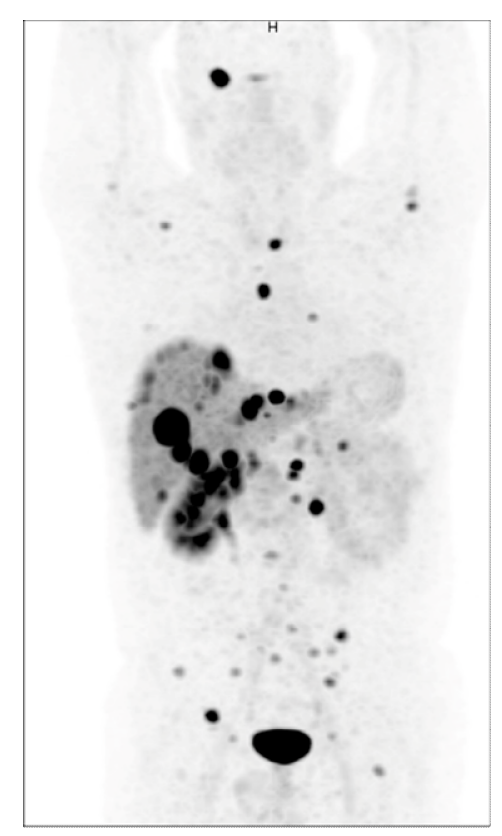

Figure 2 : Examen PET après administration de ${ }^{68}$ Ga-DOTATATE chez un patient souffrant d'une tumeur neuro-endocrine métastatique. On visualise de très nombreux foyers, qui représentent des lésions exprimant fortement les récepteurs à la somatostatine. Le traceur est éliminé par les reins, et aucun autre organe n'accumule le traceur de façon significative. Le ${ }^{177}$ Lu-DOTATATE, émetteur beta- présentera exactement la même distribution, permettant donc une irradiation spécifique et à forte dose des lésions métastatiques. 
Il existe enfin une approche qui s'appuie sur un particularisme anatomique. Le foie est doublement perfusé, par le système artériel hépatique, et par la veine porte. Cette dernière contribue à la majorité de l'afflux sanguin du foie. Certaines tumeurs hépatiques, primitives (hépatocarcinome) ou secondaires (métastases), présentent pour particularité une perfusion artérielle très nettement augmentée. On peut alors cathétériser de façon sélective l'artère hépatique ou une de ses branches, et injecter des microsphères marquées à $1^{90}$ Y. Ces microsphères, dont la taille est telle qu'elles s'embolisent dans le lit vasculaire et ne franchissent pas l'obstacle hépatique, s'accumulent ainsi dans les lésions tumorales qui se trouvent irradiées à des doses inatteignables par les techniques de radiothérapie externe (figure 3 ).

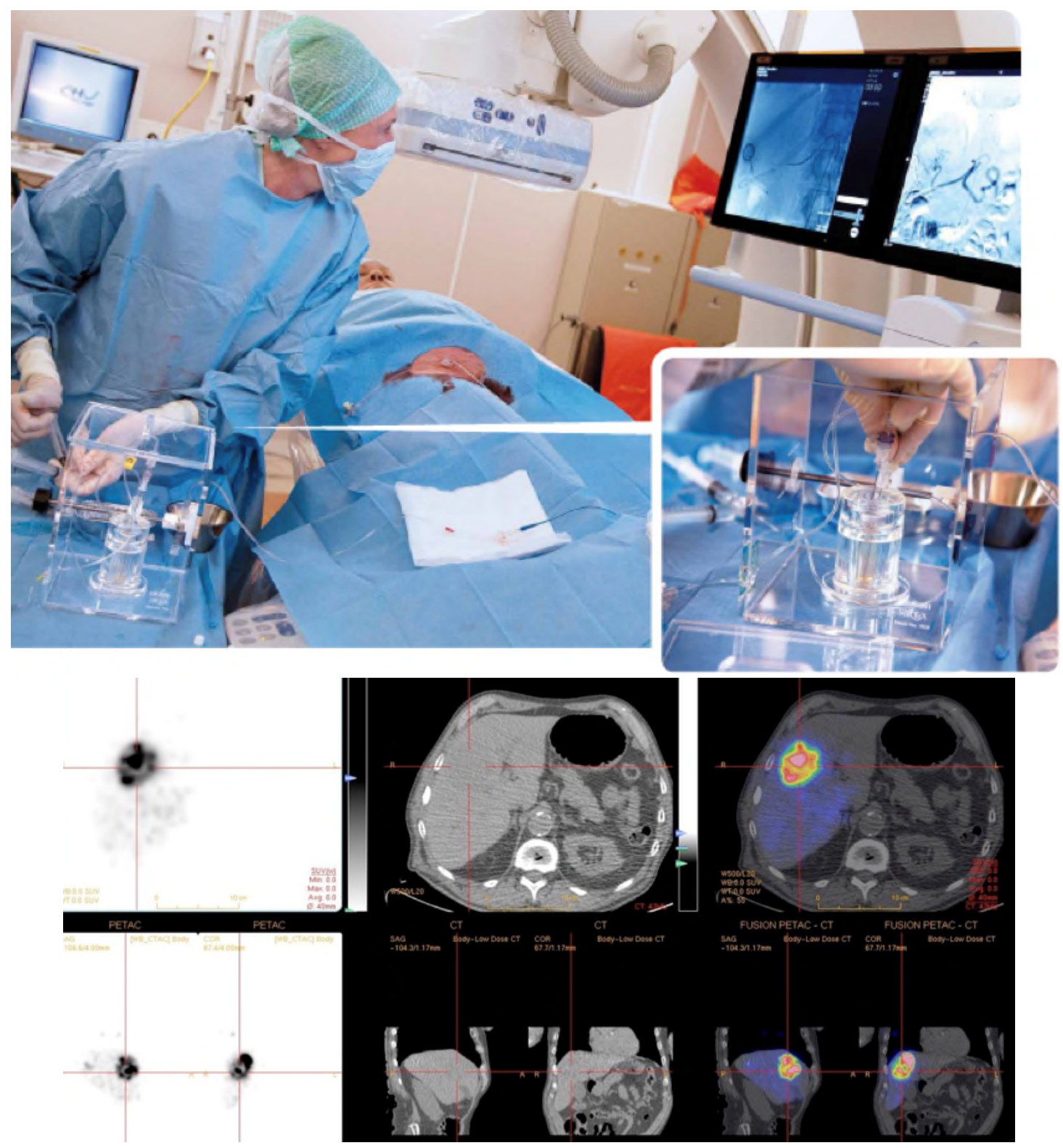

Figure 3 : Radio-embolisation artérielle hépatique sélective. Le radiologue et le médecin nucléariste travaillent en équipe pour réaliser le cathétérisme artériel et injecter les microsphères marquées à l'Y-90. L'examen PET-CT réalisé quelques heures plus tard montre que les sphères sont effectivement concentrées dans la tumeur, délivrant des doses qui peuvent atteindre plusieurs centaines de Gy. 
Les approches évoquées ici ont toutes une place dans l'armamentarium thérapeutique, mais le tableau doit être brossé de manière objective. Comme pour tout traitement, la sélection des malades est un élément clé. Il est capital d'identifier les patients les plus susceptibles de bénéficier de ce type de traitement, en fonction de l'évolution de la maladie et des autres armes disponibles. Tous ces traitements présentent des effets secondaires, dont la sévérité, la fréquence, et la prédictibilité est variable.

\section{Dosimétrie}

En contraste avec la radiothérapie externe, cet aspect est resté longtemps négligé mais est actuellement en plein essor. Le but est de déterminer de manière aussi précise que possible, l'activité à administrer à chacun des patients, pour optimiser la marge thérapeutique. La dose à la cible doit être la plus élevée, et la dose aux tissus sains, la plus faible possible. Actuellement, la plupart des traitements sont administrés suivant des schémas standardisés, mais de nombreuses études sont en cours, visant à modéliser la dosimétrie et adapter les traitements sur base individuelle. Les obstacles méthodologiques sont nombreux, et les radiopharmaciens et les physiciens médicaux jouent un rôle moteur essentiel dans ces recherches (3).

\section{Radioprotection}

Toutes les mesures doivent être prises afin de protéger la population de l'exposition liée aux utilisations thérapeutiques des rayonnements ionisants. Lorsque le traitement entraine chez le patient un débit de dose supérieur à un seuil (20 uSv/h à $1 \mathrm{~m} \mathrm{du}$ patient), il est réalisé lors d'une hospitalisation, dans un environnement adapté. Lorsque le traitement est réalisé en externe, des consignes sont transmises de manière à réduire l'exposition du public, en particulier les enfants et les femmes enceintes, plus sensibles à l'effet nocif des rayonnements. Selon les cas, on recommandera l'éviction totale ou tout simplement d'éviter les contacts prolongés et rapprochés. En outre, lorsque le radionucléide thérapeutique se retrouve dans les urines par exemple, des mesures spécifiques doivent également être prises afin d'éviter toute contamination. Les mesures de radioprotection sont le plus souvent simples, aisées à appliquer et limitées dans le temps.

\section{Références}

1. Parker C, and ALSYMPCA Investigators. Alpha emitter radium-223 and survival in metastatic prostate cancer. N Engl J Med. 2013 Jul 18;369(3):213-23.

2. Strosberg J, and NETTER-1 Trial Investigators. Phase 3 Trial of (177)Lu-Dotatate for Midgut Neuroendocrine Tumors. N Engl J Med. 2017 Jan 12;376(2):125-135.

3. Ljungberg M, Sjögreen Gleisner K. Personalized Dosimetry for Radionuclide Therapy Using Molecular Imaging Tools. Biomedicines. 2016 Nov 15;4(4) 